\title{
Cardiovascular complications according to severity of renal artery stenosis based on Doppler ultrasound
}

Łukasz Artyszuk, Bartosz Symonides, Zbigniew Gaciong, Cezary Szmigielski

Department of Internal Medicine, Hypertension and Vascular Diseases, Medical University of Warsaw, Poland

Submitted: 7 October 2020

Accepted: 28 December 2020

Arch Med Sci

DOI: https://doi.org/10.5114/aoms/132002

Copyright $\odot 2021$ Termedia \& Banach

\section{Abstract}

Introduction: The interactions between atherosclerotic renal artery stenosis, independently of severity, and cardiovascular risk, and mortality, are complex and have not been fully researched. The aim of this study was the assessment of the risk of cardiovascular events and mortality in patients with haemodynamically non-significant (NS-RAS) and significant renal artery stenosis (S-RAS) diagnosed with ultrasonography.

Material and methods: The study group consisted of all consecutive patients $(n=2059)$ who underwent Doppler ultrasound of the renal arteries during a 4-year period. The patients were divided, according to the renal aortic ratio (RAR), into the following groups: S-RAS (RAR $\geq 3.5$ ), NS-RAS $(1<$ RAR < 3.5$)$, and normal RAR (control group; RAR $\leq 1)$. The risk of cardiovascular events and death was estimated using Cox's proportional hazard model, including severity of RAS, age, and gender, based on the data from the National Health Fund on causes of hospitalization, deaths, and statistics on percutaneous coronary angioplasty procedures.

Results: Significant renal artery stenosis was found in 112 patients (5.4\%), NS-RAS in 313 patients (15.2\%), and 1634 patients (79.4\%) were qualified to the control group. The NS-RAS group had an increased risk of stroke $(7.0 \%$ vs. $3.0 \%, \mathrm{HR}=1.77, p=0.032)$; S-RAS patients were at increased risk of heart failure $(16.1 \%$ vs. $5.2 \%, \mathrm{HR}=2.19, p=0.002)$ and death $(19.6 \%$ vs. $4.3 \%, \mathrm{HR}=3.08$, $p<0.001)$

Conclusions: The presence of haemodynamically non-significant renal artery stenosis is an indicator of systemic atherosclerotic changes in vital organs and an important cardiovascular risk factor for stroke.

Key words: renal artery stenosis, cardiovascular risk, colour Doppler ultrasound, renovascular hypertension, atherosclerosis.

\section{Introduction}

Atherosclerotic renal artery stenosis (ARAS) is one of the most common causes of secondary hypertension [1-5]. Atherosclerosis is a systemic disease, and it usually develops in various organ systems. Atherosclerosis leading to ARAS, is the most common primary disease of the renal arteries [6]. In a clinical setting, ARAS, hypertension, and ischaemic complications, are variously combined $[7,8]$. The interactions between ARAS, cardiovascular risk, and mortality are complex and not fully researched [6]. The concomitant presence of hypertension and renal ischaemia constitutes high cardiovascular risk [7, 8]. Atherosclerotic renal

\author{
Corresponding author: \\ tukasz Artyszuk \\ Department of Internal \\ Medicine, Hypertension and \\ Vascular Diseases \\ Medical University of Warsaw \\ 1a Banach St \\ 02-097 Warsaw, Poland \\ E-mail: \\ lukasz.artyszuk@wum.edu.pl
}


artery stenosis is also part of a significant financial burden on any healthcare system [9]. Significant ARAS is recognized when there is over $60 \%$ narrowing of the arterial lumen [10]. However, despite optimal pharmacological management or invasive intervention, patients with significant ARAS have a higher risk of acute cardiovascular episodes than individuals without renal stenosis. This group is characterized by more frequent occurrence of heart failure (HF), myocardial infarction (MI), stroke and death for any reason [11-23]. However, so far, few studies have included patients with non-significant renal artery stenosis (NS-RAS), and none of the reviewed studies included NS-RAS diagnosed by ultrasound. Some authors concluded that NS-RAS may increase cardiovascular risk and mortality, but only a limited spectrum of cardiovascular events was assessed [11, 24].

The main aim of the study was to assess retrospectively the cardiovascular events in patients with non-significant (NS-RAS) and significant (S-RAS) renal artery stenosis when compared to patients without renal artery stenosis. The RAS narrowing was diagnosed by colour Doppler ultrasound (CDU). In the study, we analysed the significance of diagnosing NS-RAS for cardiovascular risk.

\section{Material and methods}

All consecutive patients $(n=2113)$ who underwent CDU (from 3 January 2005 to 22 December 2008) in a university hospital were initially qualified. The study included persons with CDU visualization of the renal arteries on both sides, or with an artery visible to one of the kidneys, as well as patients with renal artery visualized to the solitary kidney.

The exclusion criteria were as follows: kidney transplantation, previous invasive treatment of abdominal aortic aneurysm and/or RAS. The patients who qualified for the study were divided into 2 groups depending on the RAR result. During qualification, significant asymmetry of the kidney length (difference equal to or greater than $15 \mathrm{~mm}$ ) and failure to visualize one of the renal arteries were taken into account. An artery that could not be visualized on the side of a significantly smaller kidney was classified as an occluded artery. The S-RAS group included patients with RAR greater than or equal to 3.5 in at least one renal artery and patients with ultrasound features of an occluded artery. Patients with RAR above 1 but less than 3.5, in at least one renal artery, were included in the NS-RAS group. The control group consisted of patients with RAR less than or equal to 1 in both renal arteries [25].

During the qualification for individual groups, basic demographic data were collected from the CDU reports. From the National Health Fund (NHF), statistics related to the reasons for hospi- talization and percutaneous coronary angioplasty (PTCA) were obtained (available information about events until 31 December 2013). The data gathered included the following: dates of hospitalization and the number of the Diagnosis-Related Group (DRG) to which the patient was qualified, as well as a list of medical procedures in the International Classification of Procedures (ICD-9). The data received from the NHF also included mortality reports. The analysis of NHF data provided details about the following reasons for hospitalization: heart failure (HF), acute coronary syndrome (ACS), and stroke. The first reported hospitalization for each reason was analysed for every patient. In addition, medical procedures such as PTCA were included. Cardiovascular events were classified based on the reasons for hospitalization. Heart failure was identified by DRG codes: E52, E53, and E54, ACS: E10, E11, E12, E13, E14, E16, E17 and E18, stroke: A48, A49, and A50. PTCA procedures were classified according to the ICD 9 and were characterized by the codes 36.091, 00.661, and 00.66.

\section{Statistical analysis}

All results were evaluated for normality of distributions with the Shapiro-Wilk test. The mean age and parameters from the Doppler study were compared by the Kruskal-Wallis test, and post-hoc analysis was carried out with the Mann-Whitney test due to abnormal distribution. Quantitative variables were analysed using the chi-square test for all groups, followed by pairs analysis using the Bonferroni correction. The survival function for individual endpoints in the subgroups was determined using the Kaplan and Meyer method. The Cox proportional hazard analysis was conducted in individual RAS subgroups, corrected for age and gender. Mortality analysis was censored right-sided at the end of the study, the remaining endpoints at the end of the study or at the death of the patient. The significance level was assumed to be $p<0.05$. Statistical calculations were performed using the R package (version 3.0.2, R-core Team, R Foundation for Statistical Computing, Vienna, Austria, https://www.r-project.org).

The Bioethics Committee at the Medical University of Warsaw issued a statement on 18 October 2011, number KBO/40/11, and approved the study.

\section{Results}

A total of 2059 patients qualified for the study (Figure 1); 1016 women and 1043 men, median age was 54 years (IQR 37-66). Significant stenosis or obstruction of the renal artery (S-RAS) was 


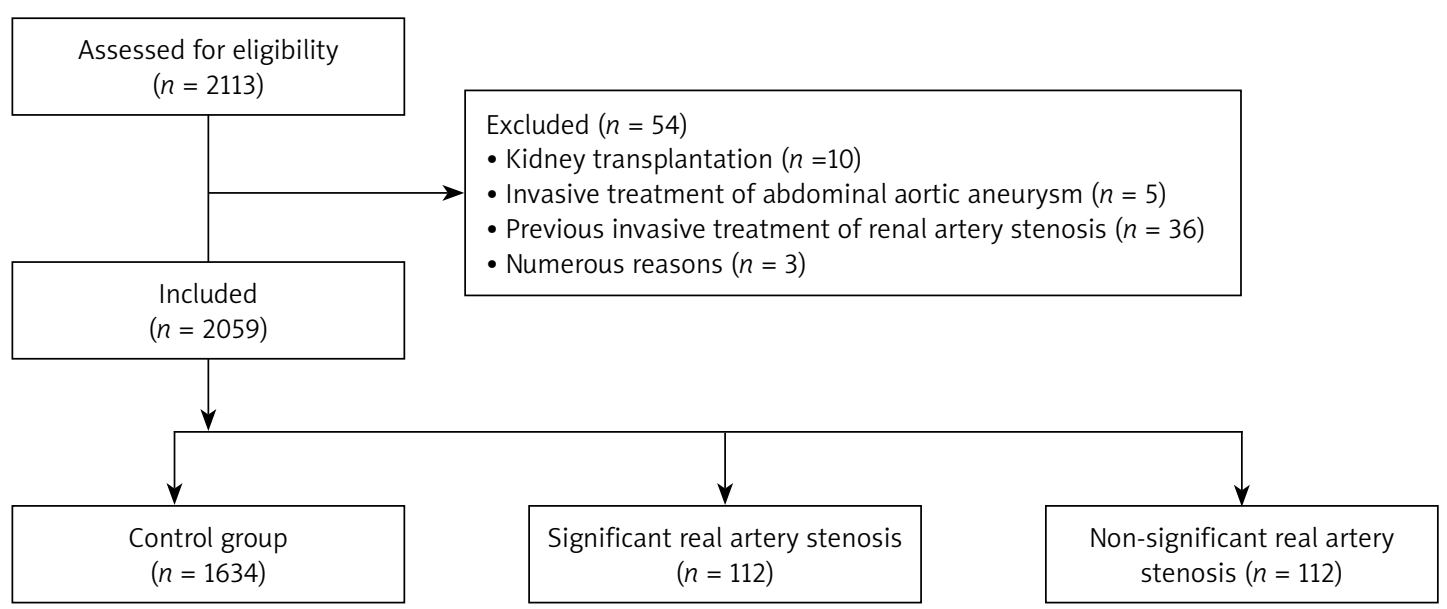

Figure 1. Qualification for the study

Table I. Renal artery stenosis in the study groups

\begin{tabular}{|c|c|c|c|c|c|c|}
\hline Patients & $\begin{array}{c}\text { Male/Female, } \\
n(\%)\end{array}$ & $\begin{array}{l}\text { Median age } \\
\text { [years] (IQR) }\end{array}$ & $\begin{array}{c}\text { Unilateral stenosis, } \\
n(\%)\end{array}$ & $\begin{array}{c}\text { Bilateral stenosis, } \\
n(\%)\end{array}$ & $\begin{array}{c}\text { One kidney, } \\
n(\%)\end{array}$ & $\begin{array}{l}\text { Occlusion, } \\
n(\%)\end{array}$ \\
\hline S-RAS & $45 / 67^{\star *}(40 / 60)$ & $60.5^{*}(51-70)$ & $68(60.7)$ & $15(13.4)$ & $2(1.8)$ & $27(24.1)$ \\
\hline NS-RAS & $111 / 202^{*}(35 / 65)$ & $62.5^{*}(52-72)$ & $146(46.6)$ & $157(50.2)$ & $10(3.2)$ & 0 \\
\hline Control & $887 / 747(54 / 46)$ & $51(33-63)$ & 0 & 0 & $45(2.8)$ & 0 \\
\hline
\end{tabular}

found in 112 people (5.4\%), while in 313 patients $(15.2 \%)$ renal artery stenosis was estimated as NS-RAS, and in 1634 patients (79.4\%) there was no evidence of stenosis of the renal artery (control group). The detailed characteristics of the groups are presented in Table I.

The NHF data on the causes of hospitalization recorded 351 hospitalizations due to cardiovascular events, such as HF (133 events), ACS (111), and stroke (80).

Patients with NS-RAS, compared with the control group, were characterized by more frequent hospitalizations due to stroke. In the NS-RAS group hospitalizations because of stroke were found in 22 patients, and in the control group there were 49 hospitalizations (7.0 \% vs. $3.0 \%$; HR = 1.77; 95\% Cl: 1.05-2.97; $p=0.032$ ) (Figure 2).

Analysis of the remaining causes of hospitalization showed no statistically significant differences between the NS-RAS group and the control group for HF 25 vs. 85 hospitalizations (8.0\% vs. 5.2\%; $\mathrm{HR}=0.92$; 95\% Cl: 0.59-1.46; NS), and for ACS 24 vs. 74 hospitalizations (7.7\% vs. $4.5 \%$; $\mathrm{HR}=1.24$; 95\% Cl: 0.78-2.00; NS) (Figure 2).

In the group of patients with S-RAS, a higher risk of hospitalizations due to HF was found when compared to the control group. In the S-RAS group 18 hospitalizations were secondary to HF, and in the control group there were 85 hospitalizations because of HF (16.1\% vs. $5.2 \%$; HR = 2.19; and 95\% Cl: 1.32-3.65; $p=0.002$ ) (Figure 2).

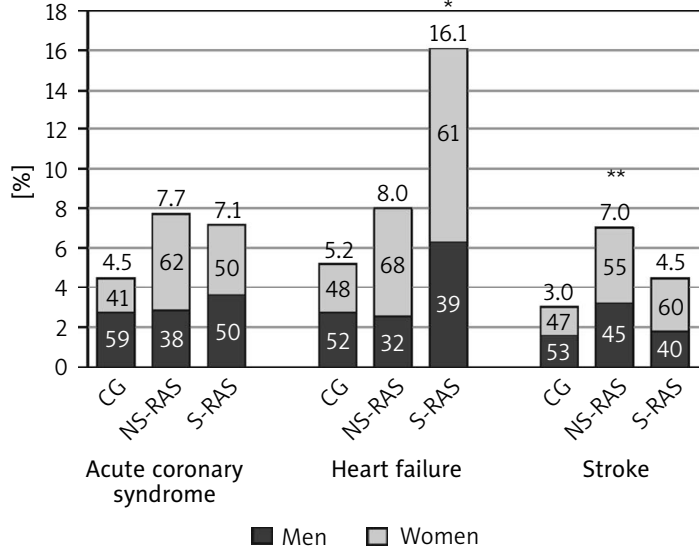

Figure 2. Comparison of reasons for hospitalization in the group with non-significant renal artery stenosis (NS-RAS) and significant renal artery stenosis (S-RAS). CG - control group. ${ }^{\star} P<0.01,{ }^{* *} p<0.05$

Analysis of the remaining causes of hospitalization showed no significant differences between the S-RAS group and the control group, including stroke 5 vs. 49 (4.5\% vs. $3.0 \%$; $\mathrm{HR}=1.09 ; 95 \% \mathrm{Cl}$ : $0.43-2.74$; NS) and ACS 8 vs. 74 (7.1\% vs. $4.5 \%$; $\mathrm{HR}=1.17$; 95\% Cl: 0.56-2.44; NS) (Figure 2).

Among the studied patients, PTCA procedures were performed in 79 individuals. There were no significant differences when comparing the frequency of PTCA in the S-RAS group (4 procedures) and the NS-RAS group (20 procedures) to 52 procedures in the control group (3.6\% vs. 3.2\%; 


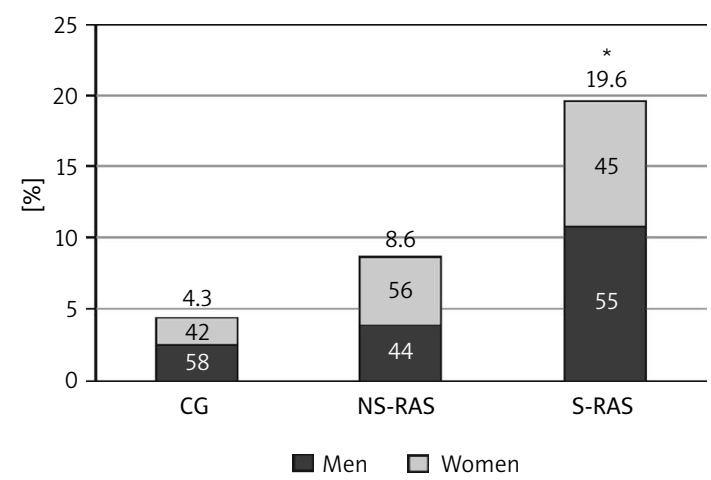

Figure 3. Percentage of deaths in the study groups. CG - control group, NS-RAS - non-significant renal artery stenosis, S-RAS - significant renal artery stenosis. ${ }^{*} P<0.05$

$\mathrm{HR}=0.84 ; 95 \% \mathrm{Cl}: 0.30-2.35 ; \mathrm{NS} ; 6.4 \%$ vs. $3.2 \%$; $\mathrm{HR}=1.62 ; 95 \% \mathrm{Cl}: 0.95-2.77 ; p=0.07)$.

The data from the NHF showed the occurrence of 120 deaths among the studied patients. Of these, 71 deaths occurred in the control group, 22 deaths occurred in the S-RAS group, and 27 deaths occurred in the NS-RAS group. The comparison with the control group, adjusted for age and gender, showed a statistically significantly higher death rate in the S-RAS group when compared to the control group (19.6\% vs. $4.3 \%$; HR $=3.08 ; 95 \%$ $\mathrm{Cl}: 1.91-4.98 ; p<0.001)$. However, no significant difference was found between the NS-RAS group and the control group ( $8.6 \%$ vs. $4.3 \%$; $H R=1.18$; 95\% Cl: 0.75-1.86; NS) (Figure 3). Over the study period, survival analysis showed significantly higher mortality in the S-RAS group compared to the control group and no significant difference in the NS-RAS group (Figure 4).

\section{Discussion}

In this study nearly $20 \%$ of patients showed abnormal renal artery Doppler parameters. Significant RAS was diagnosed in $5.4 \%$ of patients, and non-significant RAS was identified in $15.2 \%$ of patients. To date, the frequency of RAS based on CDU has not been estimated in such a large group of patients in the local population. In the 2 largest studies reviewed for this discussion the incidence of RAS differed significantly. In the De Mast and Beutler meta-analysis, based on 40 papers $(n=15,879)$, the authors found the presence of stenosis in $15.4 \%$ of patients [26], and Crowley et al. found RAS in $6.3 \%$ among 14,172 subjects [27]. The incidence of NS-RAS in this study was estimated at $15.1 \%(n=313)$ based on an RAR over 1 and below 3.5. In the available literature, we found no other study that estimated the incidence of NS-RAS in a large patient group based on CDU. Studies evaluating renal artery stenosis diagnosed with arteriography have shown the oc-

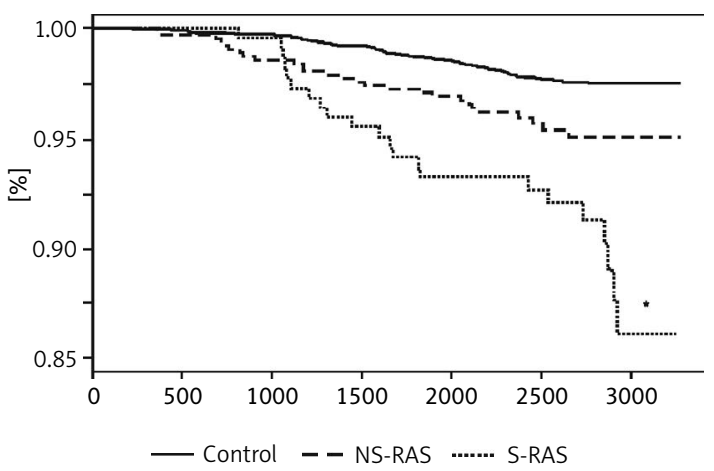

Figure 4. Risk of death depending on the degree of renal artery stenosis according to survival function analysis using Kaplan-Maier estimator. NS-RAS - non-significant renal artery stenosis, S-RAS - significant renal artery stenosis. ${ }^{*} P<0.05$

currence of NS-RAS at a similar frequency as in our study $[15,28]$.

In our study, the incidence of cardiovascular events was assessed based on data on the causes of hospitalization obtained from the NHF. Heart failure was the most common cause of hospitalization among all patients qualified for the study and was diagnosed in 133 people (6.5\%). The more frequent occurrence of heart failure in patients with RAS has been confirmed in previous studies [17, 24].

In our study, there was a statistically significantly higher risk of hospitalizations due to stroke among NS-RAS patients compared to the control group; such a relationship was not found in the S-RAS group. Kawarada et al. assessed the frequency of a history of stroke, new stroke, or transient ischaemic attack (TIA) episodes in a group with RAS diagnosed based on CDU [16]. Cerebrovascular accidents (CVA) were more common in the RAS group (23.7\% vs. $11.3 \%)$. Kalra et al. in their study presented the incidence of CVA in $36.9 \%$ of people with RAS vs. $12 \%$ without stenosis [17]. This discrepancy is most likely due to the fact that the study included not only the new incidents, but also a history of stroke or TIA.

The cause of high prevalence of CVA among patients with RAS is the coexistence of atherosclerotic plaques in many arterial locations, including carotid arteries. However, no studies have yet been identified to assess the occurrence of stroke in patients with haemodynamically non-significant RAS. Demonstration of more frequent stroke episodes in the NS-RAS population in this study have practical clinical significance. It is worth discussing the use in this group of clinical management similar to that used in patients with asymptomatic carotid stenosis, including a statin to achieve LDL levels below $70 \mathrm{mg} / \mathrm{dl}$ and an earlier start of antiplatelet therapy [10].

In our group, 111 hospitalizations due to ACS were recorded, which occurred in $5.4 \%$ of the pa- 
tients. Despite more frequent hospitalizations for ACS, in patients with NS-RAS and S-RAS the difference was not statistically significant. In contrast to this finding, previous studies have shown a significantly higher incidence of ACS in RAS patients [29]. In our study, 79 PTCA procedures were performed. There was a tendency to perform more PTCA in the NS-RAS group compared to the control group. However, the difference was not statistically significant. The results of older studies are not conclusive for assessment of the risk of $\mathrm{MI}$ or the need for PTCA for patients with RAS [29-31].

In the whole observation period of the 5 years of follow-up, a total of 125 deaths (6.1\%) were recorded in the entire study group. A statistically higher risk of death was found in patients with S-RAS, and in the NS-RAS group there was a trend towards higher mortality compared to the control group, but this was not statistically significant. The results of our study confirm the high mortality of patients with RAS. According to the Medicare registry, an analysis of $5 \%$ of random data gathered in a 2-year period showed a higher mortality rate in patients with RAS [17]. Also, the latest randomized studies showed similar mortality in both pharmacologically and invasively treated groups: in STAR within 2 years $8 \%$, in ASTRAL within 5 years $25.9 \%$, in CORAL within 3.5 years $14.9 \%$ [32-34]. In the cited studies, despite the treatment, the mortality was high, similarly to previous observations. Unfortunately, to date, there have been few studies assessing the risk of death in patients with haemodynamically insignificant RAS. In a study by Zanoli et al., the authors in a 4.5-year follow-up showed greater cardiovascular mortality in patients with RAS $10-70 \%$ compared to RAS < $10 \%$ (12.7\% vs. $5.6 \%$ ) [24]. In other study, Safak et al. showed that mortality was significantly higher in patients with N-RAS below $50 \%$ than in the control group, similarly to that in the group with RAS [11]. Although our study did not show significantly increased mortality in the NS-RAS group, there is a probability that, due to the tendency for higher mortality in this group, statistical significance could be expected in a longer follow-up.

Our study presents some typical limitations of a retrospective analysis of health care reimbursement data. Information about the cardiac episodes and the procedures came from the NHF database and corresponded to the codes of procedures performed by healthcare providers, as well as the DRG data. Due to the limitations of the database, the authors did not have access to the listed medical data, such as medical history and pharmacological treatment. Another limitation is the inability to differentiate RAS aetiology in the CDU study. There is a risk of potential bias and errors in the reporting of some diagnoses or procedures. The non-randomized design is another limitation.

In conclusion, patients with Doppler ultrasound features of haemodynamically insignificant renal artery stenosis had an increased risk of stroke. Patients with significant stenosis of the renal artery, confirmed by ultrasound, had higher mortality and higher risk of worsening heart failure, compared to patients without stenosis. There were no significant differences in the frequency of hospitalization due to acute coronary syndrome and percutaneous coronary interventional procedures, in comparison with patients without renal artery stenosis.

The presence of haemodynamically non-significant renal artery stenosis is an indicator of systemic atherosclerotic changes in vital organs and an important cardiovascular risk factor for stroke.

\section{Conflict of interest}

The authors declare no conflict of interest.

\section{References}

1. Berglund G, Andersson O, Wilhelmsen L. Prevalence of primary and secondary hypertension: studies in a random population sample. Br Med J 1976; 2: 554-6.

2. Danielson M, Dammstrom B. The prevalence of secondary and curable hypertension. Acta Med Scand 1981; 209: 451-5.

3. Vokonas PS, Kannel WB, Cupples LA. Epidemiology and risk of hypertension in the elderly: the Framingham Study. J Hypertens Suppl 1988; 6: S3-9.

4. Anderson GH Jr, Blakeman N, Streeten DH. The effect of age on prevalence of secondary forms of hypertension in 4429 consecutively referred patients. J Hypertens 1994; 12: 609-15.

5. Shukla AN, Madan TH, Jayaram AA, et al. Prevalence and predictors of renal artery stenosis in patients undergoing peripheral and coronary angiography. Int Urol Nephrol 2013; 45: 1629-35.

6. Zoccali C, Mallamaci F, Finocchiaro P. Atherosclerotic renal artery stenosis: epidemiology, cardiovascular outcomes, and clinical prediction rules. J Am Soc Nephrol 2002; 13 Suppl 3: S179-83.

7. Safian RD, Textor SC. Renal-artery stenosis. New Engl J Med 2001; 344: 431-42.

8. Textor SC. Renal Arterial Disease and Hypertension. Med Clin North Am 2017; 101: 65-79.

9. Wierzejska E, Giernas B, Lipiak A, Karasiewicz M, Cofta M, Staszewski R. A global perspective on the costs of hypertension: a systematic review. Arch Med Sci 2020; 16: 1078-91.

10. Aboyans V, Ricco JB, Bartelink MEL, et al. 2017 ESC guidelines on the diagnosis and treatment of peripheral arterial diseases, in collaboration with the European Society for Vascular Surgery (ESVS): Document covering atherosclerotic disease of extracranial carotid and vertebral, mesenteric, renal, upper and lower extremity arteriesEndorsed by: the European Stroke Organization (ESO)The Task Force for the Diagnosis and Treatment of Peripheral Arterial Diseases of the European Society 
of Cardiology (ESC) and of the European Society for Vascular Surgery (ESVS). Eur Heart I 2018; 39: 763-816.

11. Safak E, Wilke C, Derer W, et al. Long-term follow-up of patients with atherosclerotic renal artery disease. J Am Soc Hypertens 2013; 7: 24-31.

12. Messerli FH, Bangalore $\mathrm{S}$, Makani H, et al. Flash pulmonary oedema and bilateral renal artery stenosis: the Pickering syndrome. Eur Heart J 2011; 32: 2231-5.

13. Conlon PJ, Little MA, Pieper K, Mark DB. Severity of renal vascular disease predicts mortality in patients undergoing coronary angiography. Kidney Int 2001; 60: 1490-7.

14. Gray BH, Olin JW, Childs MB, Sullivan TM, Bacharach JM. Clinical benefit of renal artery angioplasty with stenting for the control of recurrent and refractory congestive heart failure. Vasc Med 2002; 7: 275-9.

15. Dzielinska Z, Januszewicz A, Demkow M, et al. Cardiovascular risk factors in hypertensive patients with coronary artery disease and coexisting renal artery stenosis. J Hypertens 2007; 25: 663-70.

16. Kawarada O, Yokoi Y, Morioka N, Takemoto K. Renal artery stenosis in cardio-and cerebrovascular disease: renal duplex ultrasonography as an initial screening examination. Circ J 2007; 71: 1942-7.

17. Kalra PA, Guo H, Kausz AT, et al. Atherosclerotic renovascular disease in United States patients aged 67 years or older: risk factors, revascularization, and prognosis. Kidney Int 2005; 68: 293-301.

18. Conlon PJ, Athirakul K, Kovalik E, et al. Survival in renal vascular disease. J Am Soc Nephrol 1998; 9: 252-6.

19. Losito A, Errico R, Santirosi P, Lupattelli T, Scalera GB, Lupattelli L. Long-term follow-up of atherosclerotic renovascular disease. Beneficial effect of ACE inhibition. Nephrol Dial Transplant 2005; 20: 1604-9.

20. Textor SC. Current approaches to renovascular hypertension. Med Clin North Am 2009; 93: 717-32.

21. Kawarada O, Yasuda S, Noguchi T, Anzai T, Ogawa H. Renovascular heart failure: heart failure in patients with atherosclerotic renal artery disease. Cardiovasc Interv Ther 2016; 31: 171-82.

22. Khan AR, Sheikh M, Kaw D, Cooper CJ, Khouri SJ. Prevalence and factors associated with left ventricular remodeling in renal artery stenosis. J Am Soc Hypertens 2014; 8: 254-61.

23. Imori Y, Akasaka T, Ochiai T, et al. Co-existence of carotid artery disease, renal artery stenosis, and lower extremity peripheral arterial disease in patients with coronary artery disease. Am J Cardiol 2014; 113: 30-5.

24. Zanoli L, Rastelli S, Marcantoni C, et al. Non-hemodynamically significant renal artery stenosis predicts cardiovascular events in persons with ischemic heart disease. Am J Nephrol 2014; 40: 468-77.

25. Drelich-Zbroja A. Standardy badań ultrasonograficznych Polskiego Towarzystwa Ultrasonograficznego - aktualizacja. Badanie ultrasonograficzne tętnic nerkowych. J Ultrason 2014; 14: 297-305.

26. de Mast Q, Beutler JJ. The prevalence of atherosclerotic renal artery stenosis in risk groups: a systematic literature review. J Hypertens 2009; 27: 1333-40.

27. Crowley JJ, Santos RM, Peter RH, et al. Progression of renal artery stenosis in patients undergoing cardiac catheterization. Am Heart J 1998; 136: 913-8.

28. Harding MB, Smith LR, Himmelstein SI, et al. Renal artery stenosis: prevalence and associated risk factors in patients undergoing routine cardiac catheterization. J Am Soc Nephrol 1992; 2: 1608-16.

29. Burlacu A, Siriopol D, Voroneanu L, et al. Atherosclerotic renal artery stenosis prevalence and correlations in acute myocardial infarction patients undergoing primary percutaneous coronary interventions: data from nonrandomized single-center study (REN-ACS) - a single center, prospective, observational study. J Am Heart Assoc 2015; 4: e002379.

30. Ghaffari S, Sohrabi B, Siahdasht RB, Pourafkari L. Prevalence and predictors of renal artery stenosis in hypertensive patients undergoing coronary angiography. Hypertens Res 2009; 32: 1009-14.

31. Edwards MS, Craven TE, Burke GL, Dean RH, Hansen KJ. Renovascular disease and the risk of adverse coronary events in the elderly: a prospective, population-based study. Arch Intern Med 2005; 165: 207-13.

32. Wheatley K, Ives N, Gray R, et al. Revascularization versus medical therapy for renal-artery stenosis. New Eng J Med 2009; 361: 1953-62.

33. Cooper CJ, Murphy TP, Cutlip DE, et al. Stenting and medical therapy for atherosclerotic renal-artery stenosis. New Engl J Med 2014; 370: 13-22.

34. Bax L, Woittiez AJ, Kouwenberg HJ, et al. Stent placement in patients with atherosclerotic renal artery stenosis and impaired renal function: a randomized trial. Ann Intern Med 2009; 150: 840-8, W150-1. 